\title{
Essential tremor: electrophysiological and pharmacological evidence for a subdivision
}

\author{
G DEUSCHL, * C H LÜCKING,* E SCHENCK† \\ From the Department of Clinical Neurology and Neurophysiology, ${ }^{*}$ and of General Psychiatry, $\dagger$ University \\ Freiburg i. Br., West-Germany
}

\begin{abstract}
SUMMARY Forty five patients with essential tremor have been investigated by means of clinical examination, polygraphic EMG records and testing of long-latency reflexes. Clinically there were no differences between the patients, whereas the electrophysiological investigations suggested two subtypes. One group of patients may be characterised by normal long-latency reflexes and synchronous tremor bursts in antagonists or activity of the antigravity muscle alone. The second group had abnormal long-latency reflexes and reciprocal EMG activity in antagonists. It is suggested that these two groups represent distinct subgroups of essential tremor. Patients of the first group responded well to propranolol, whereas those of the second group did not.
\end{abstract}

Essential tremor is a common neurological disorder. Recent epidemiological studies have concluded that it is the most frequently occurring movement disorder. ${ }^{12}$ Although essential tremor is typically characterised by a monosymptomatic tremor mainly of the postural type, it is generally accepted that infrequently a number of associated conditions may occur. In our opinion the following criteria have to be fulfilled for the diagnosis of essential tremor: (1) tremor mainly under postural and action conditions, (2) a lack of additional severe neurologic findings or complaints (except a small number of neurologic diseases such as torticollis, dystonia, etc., which may be associated with essential tremor), (3) slow progression of tremor over years.

Essential tremor is probably not a homogenous entity and Marsden et al ${ }^{3}$ have proposed four subclasses on basis of clinical data, tremor frequency and related diseases. The present study was undertaken to investigate whether electrophysiological examination may help in further classification of essential tremor. Besides the determination of tremor frequency, the activation pattern of tremor bursts in antagonistic muscles has been analysed. In addition we studied the pattern of electrically provoked reflexes. The latter

Address for reprint requests: G Deuschl, MD, Department of Clinical Neurology and Neurophysiology, University Freiburg, Hansastr. 9, D-7800 Freiburg i. Br.

Received 15 April 1986 and in revised form 23 October 1986 Accepted 23 December 1986 investigation was done to detect pathological reflex mechanisms, which might contribute to tremor genesis. Treatment of many patients was followed up for several months and related to the diagnostic criteria. Evidence for a subdivision of essential tremor was found. Preliminary results have appeared elsewhere. ${ }^{4}$

\section{Methods and patients}

Forty five patients with essential tremor were included in this study. All of them came to neurologists with the major complaint of tremor (table 2). Diagnosis was accepted when besides tremor only minor symptoms occurred, which were present for at least 6 months without progression. Sixty per cent of the patients had a family history of tremor. Clinical testing consisted of a neurological examination and a classification and grading of tremor of the hands and arms (table 1).

The grading included a clinical rating of the amplitudes of tremor. Tremor under resting conditions was accepted, when it occurred while sitting in a comfortable chair with relaxed arms. Tremor under postural conditions was accepted if it occurred with outstretched arms. Action tremor, which occurred when performing alternating flexion/extension movements of the hand, was separated from tremor which occurred when performing finger-nose-test or finger-finger-test. Furthermore, the behaviour of tremor was rated when the mode of innervation changed from rest to postural conditions or from posture to goal-directed movements. The tremor was graded according to table 1 . If there were asymmetries in amplitude the more affected side was rated.

Polygraphic EMG recordings with surface electrodes of two or three pairs of antagonistic muscles were performed 
Table 1 Criteria for grading of tremor in hands and arms under defined conditions

Tremor under resting conditions

(tested sitting in a relaxed position with supported arms)

0 : no tremor

1: intermittent tremor, small amplitude, which can be activated by mental load

2: constant tremor with variable amplitude below $10 \mathrm{~cm}$

3 : constant tremor with amplitudes over $10 \mathrm{~cm}$ and which may not or only briefly be suppressed voluntarily.

Tremor under postural conditions

(tested by holding a water filled glass with outstretched arms)

0 : no tremor

$1:$ tremor of low amplitude without spilling water

2: moderate tremor with intermittent spilling of water

3 : tremor of large amplitude and severe spilling of water

Tremor under action conditions

A (slow alternating flexion/extension movements of hands and arms)

0 : no tremor

1 : slight tremor amplitudes

2: moderate tremor amplitudes

3: large tremor amplitudes

B (goal-directed movements: finger-finger- and finger-nose-Test)

0 : no tremor

1: slight tremor amplitudes

2: moderate tremor amplitudes

3: large tremor amplitudes

Transition among different conditions

Rest $\rightarrow$ posture

0 : suppression of tremor

1 : unchanged tremor

Posture $\rightarrow$ goal-directed movements

3 : enhanced tremor

0 : suppression of tremor

1 : unchanged tremor

3 : enhanced tremor

under resting, postural and action conditions. In all patients the frequency of the tremor at the wrist joint was measured with an accelerometer. The mode of activation of antagonists was determined with outstretched hands in pronation.

Long-latency reflexes were elicited by electrical stimulation of the median nerve at the wrist while the thumb was abducted with $10-20 \%$ of maximum force. Intensity of stimulation was chosen near motor threshold. EMG was recorded from the thenar muscles. The signal was rectified and averaged (64-256 sweeps). The normal pattern (50 normal persons) consists of an $\mathrm{H}$-reflex with a latency of $29.3 \mathrm{~ms}$ $\pm 1.8 \mathrm{~ms}$ (mean $\pm \mathrm{SD}$ ) and an LLR II at $51.8 \pm 2.8 \mathrm{~ms}^{5}$ Both hands were investigated in the patients. Efficacy of therapy was evaluated by clinical assessment. Improvement was rated on a three point scale (no improvement, moderate improvement, marked improvement).

\section{Results}

\section{Clinical data}

In all cases the tremor was of the postural type (table 2), but sometimes it occurred at rest if the patients were not completely relaxed. All the patients also exhibited action tremor of various amount. Five patients had slight resting tremor which increased during transition from resting to postural conditions. Whenever tremor persisted during goal-directed movements it was moderately or markedly suppressed during the transition. All the patients had difficulties in everyday activities, for example holding a glass, eating, writing, etc. The amount of disability was different. For the younger patients secondary impairment in social contacts due to the tremor was sometimes more prominent than the disturbances of their hands functions. Additional disorders were found in six patients (table 2), but in no case were they contrary to the diagnosis of essential tremor. From the clinical criteria the population seemed to be homogeneous.

\section{Electrophysiological data}

The electrophysiological investigation, however, suggested a division into two subgroups, termed group A and $\mathrm{B}$, differing in the mode of activation in antagonistic muscles and reflex pattern rather than in tremor frequency. In the first group (A) the frequency of tremor (table 2) was slightly higher (mean $7.5 \mathrm{~Hz}, \mathrm{SD}$ $1.3 \mathrm{~Hz}$ ) than in the second $(B$, mean $6.3 \mathrm{~Hz}$, SD $0.6 \mathrm{~Hz}$ ) due to the values of six patients who had faster frequencies between 8 and $10 \mathrm{~Hz}$.

These frequencies are in the range of physiological tremor; however, no evidence for exaggerated physiological tremor was found from clinical and laboratory investigations in these patients. Therefore they were diagnosed as essential tremor. The frequency of tremor remained constant in all the patients under postural as well as action conditions.

Three patterns of tremor bursts in antagonistic muscles were found: the activation of the antigravity muscles alone, the synchronous activation of antagonists and alternating activity in antagonists (fig $1 \mathrm{a}-\mathrm{c}$ ). 
(a)

Flex.dig.c

Ext.dig. c.

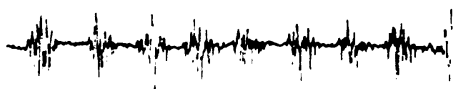

(b)

Flex. dig.c.

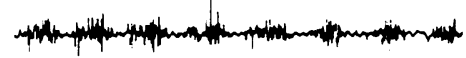

Ext. dig. C.

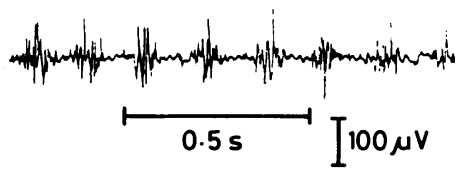

(C)

Flex. dig. c.

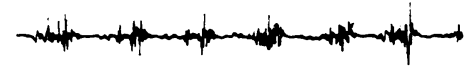

Ext. dig. c.

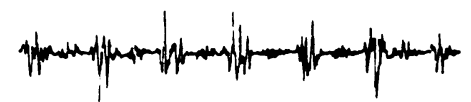

Fig 1 Electromyographic paiterns of antagonistic muscles in essential tremor. Patients with group A-ET had either tremor activity of the agonist alone (a) or synchronous bursts in antagonists (b). Patients with group B-ET had reciprocal alternating activity in antagonists $(c)$.

Type A

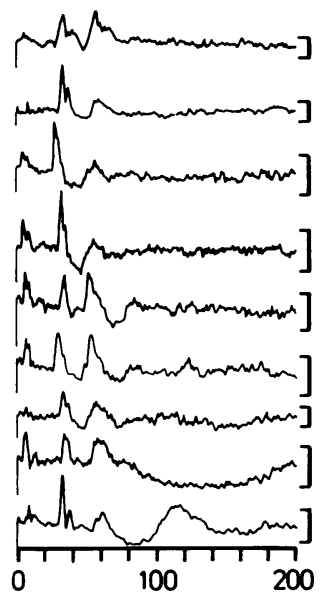

Time (ms)

Fig 2 Long latency reflexes in patients with essential tremor of group $A$ ( $n=9$ patients) and group $B(n=9$ patients $)$. At about $30 \mathrm{~ms}$ the $H$-reflex can be seen in all records. In group $B$ there is the LLRI at about $41 \mathrm{~ms}$ with pronounced amplitude and in group $A$ there is the LLR II at about $52 \mathrm{~ms}$. Vertical bars: $100 \mu \mathrm{V}$.

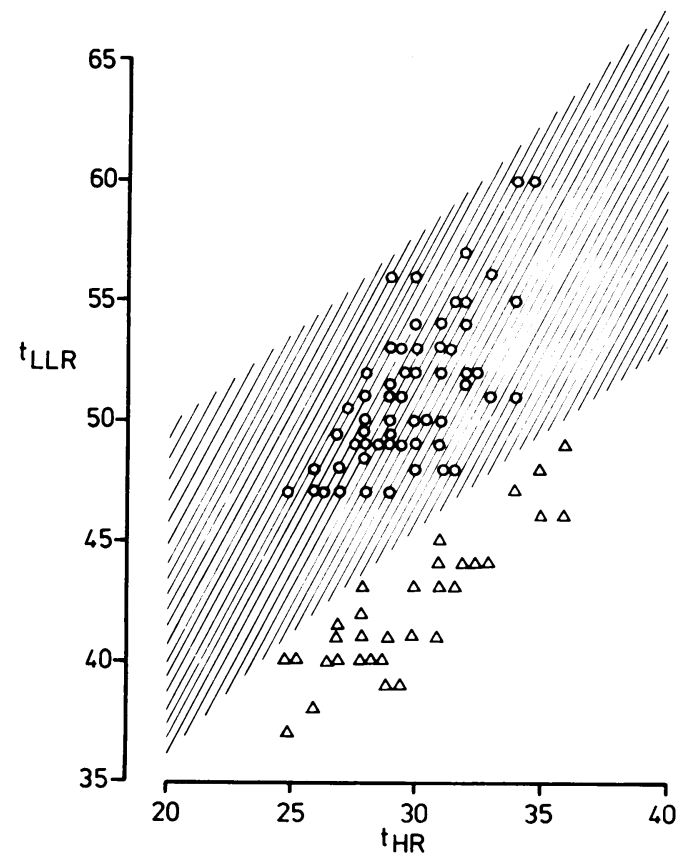

Fig 3 Long latency reflex latency ( $\left.t_{\mathrm{LLR}}\right)$ as a function of $H$-reflex latency $\left(t_{\mathrm{HR}}\right)$. Every symbol represents the values of one hand. The shaded area covers the $99 \%$ confidence region in normals. The values of group A patients $(\bigcirc)$ lie inside the normal range ( $n=29$ patients, both hands), whereas those of group B patients (A) lie clearly outside ( $n=16$ patients, both hands). Note the bimodal distribution.

Group A patients had either synchronous activation of antagonists or activity in the antigravity muscle alone. In group B patients the activation pattern was alternating. In action tremor the activation pattern was not uniform in some patients, therefore only the results under postural conditions were taken into account.

Long latency reflexes were different in the groups as well. The normal pattern of long latency reflex consists of an $\mathrm{H}$-reflex and a subsequent long latency reflex at a mean latency of $51.8 \mathrm{~ms}$ (this is called LLR II). Thirty per cent of normals also exhibited a response at about $41.3 \mathrm{~ms}$ (mean, LLR I). This is usually a very small, rather negligible reflex. In normals its amplitude is always less than $180 \%$ of baseline EMG activity. The group A patients exhibited a normal pattern with H-reflex and LLR II, whereas the group B patients had an abnormal large LLR I, which exceeded in every case $200 \%$ of baseline activity. Sometimes it was even larger than $\mathrm{H}$-reflex (fig 2). Abnormally large LLR I have been found in all group $B$ patients in both hands. In most cases with unilateral 
Table 2 Clinical and electrophysiological data of the 45 patients. For rating of tremor see table 1

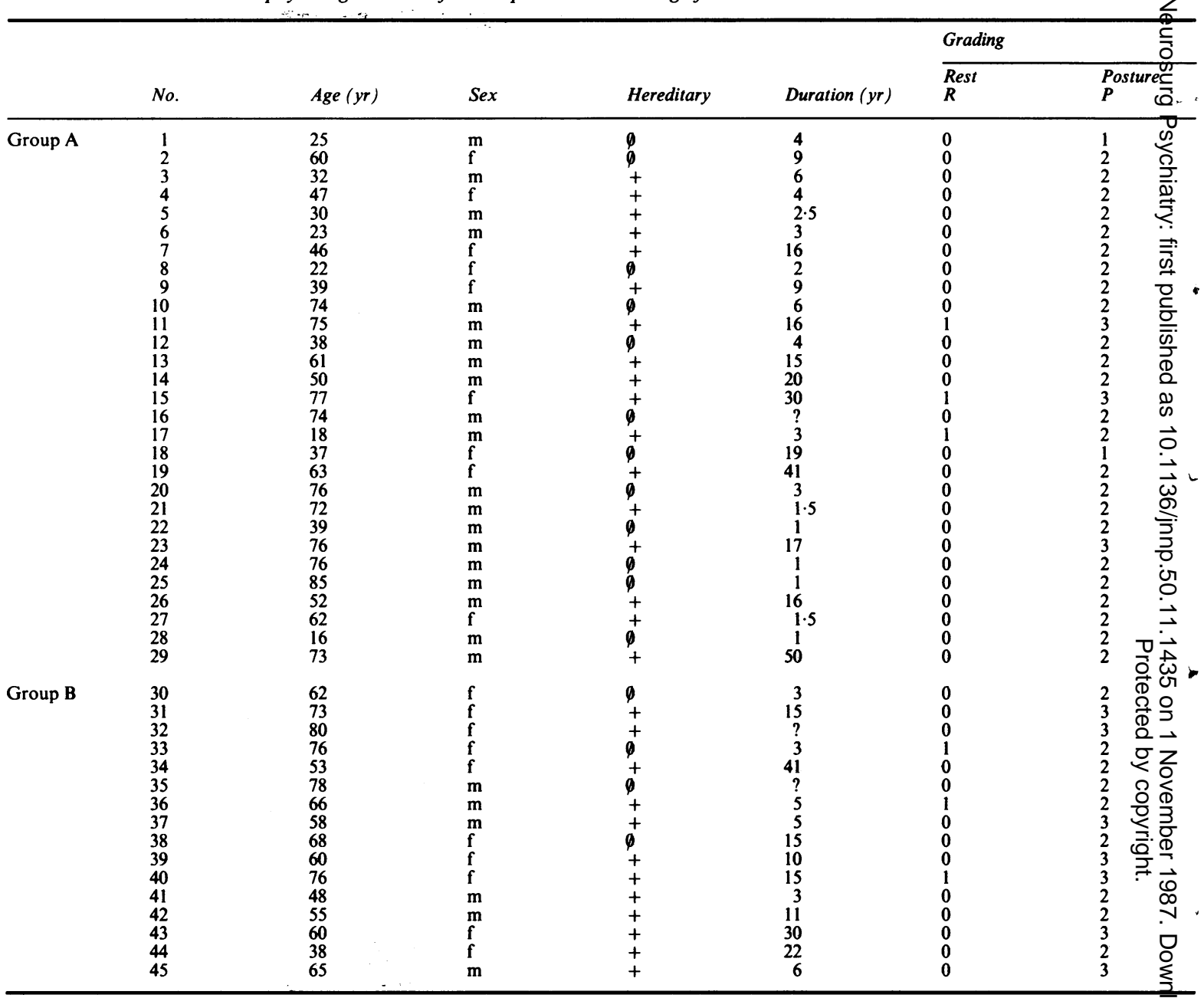

m, male; f, female; s, syndroneous; $r$, reciprocal alternating; a, agonist alone; A, alternating flexion/extension movements; B, goal-directed moveme $\overline{\text { s }}$; $\emptyset$, not applicable.

preponderance of tremor amplitudes, the LLR I amplitude was larger on the more affected side. Others, however, had no clear-cut amplitude differences despite different tremor expression. LLR II was mostly absent in these patients.

Figure 3 shows the long latency reflex latency in these two groups as a function of the H-reflex latency. The shaded area represents the confidence region which covers $99 \%$ of all normal data. With this kind of display the peripheral conduction time is eliminated and therefore the separation of the two reflexes is more pronounced. The patients of group B fall clearly outside whereas the patients of group A fit with the normal values.
Therapeutic effects

The data of therapeutic effects of different drugs are listed in table 3. Only some of the patients could be followed up. We usually started with propranolol as the drug of first choice. The dose was slowly increased up to $120 \mathrm{mg} /$ day. If any improvement was found it was further increased. The maximum dose, however, was limited in some of the elder patients by cardiovascular side effects. The therapeutic response was moderate or marked in patients of group A, whereas only two persons among the group B had a mild improvement by propranolol. If propranolol did not improve the tremor, primidone was used. Nine of the 10 patients of group B who had been given primidone 


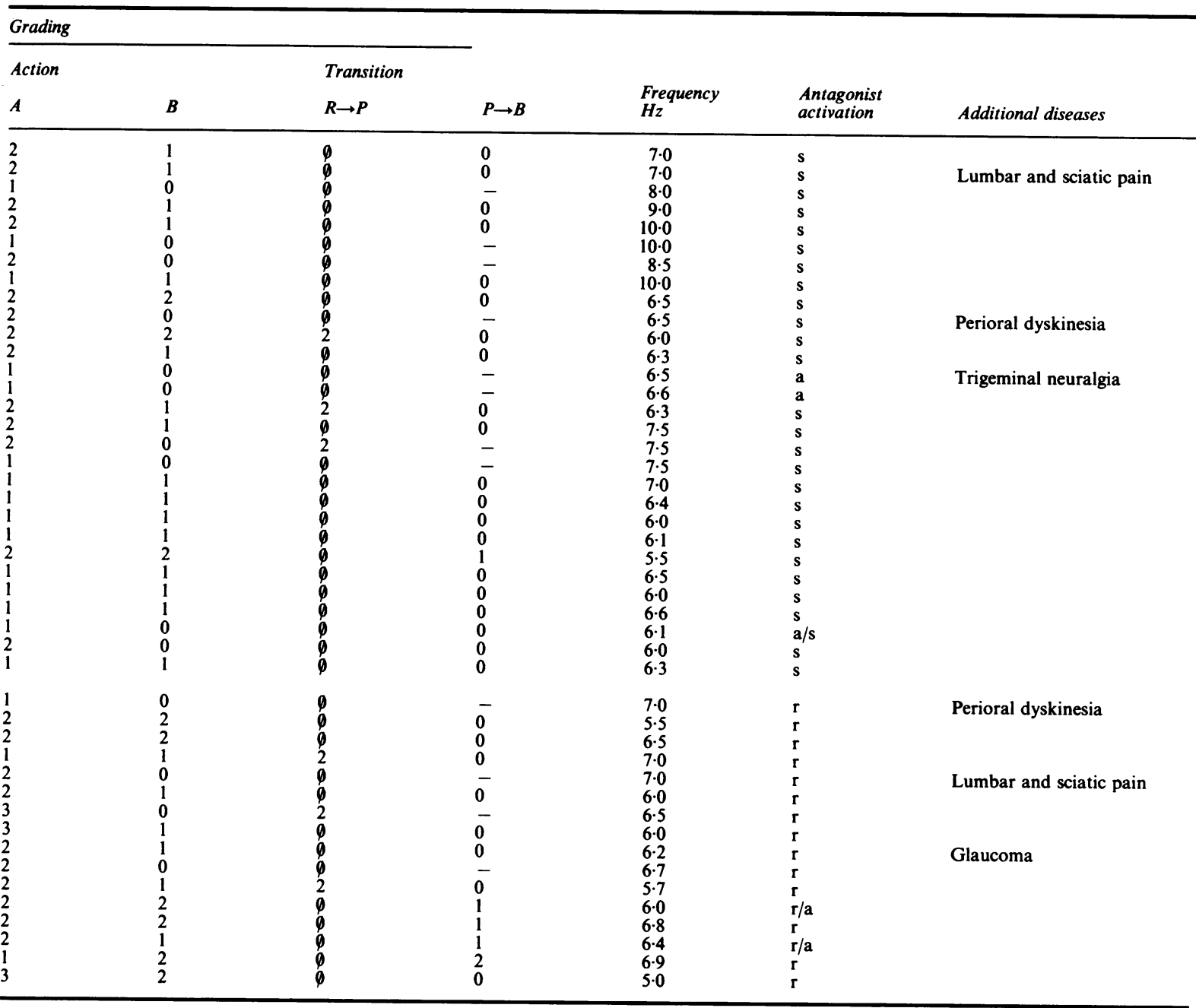

had a marked improvement after primidone $(750$ $\mathrm{mg} / \mathrm{day})$. In some of these patients the tremor was almost abolished. One patient had no improvement with either drug or in combination. Two patients were followed up for 2 years. The drug was slowly discontinued in both over several days and their tremor again became very prominent. After renewed application of primidone they improved again.

\section{Discussion}

The study presents electrophysiological data of a clinically rather homogenous group of patients with essential tremor. In agreement with a recent paper ${ }^{7}$ the six patients with faster frequencies have to be included among those with essential tremor, because their tremor could not be accepted as exaggerated physiological tremor. ${ }^{6}$ These patients had normal thyroid function and normal serum electrolytes. They had no medication, no history of alcoholism or other diseases and most of them had a family history of tremor. According to the criteria of Marsden et al $^{3}$ these six patients would belong to type I-ET, whereas the remaining majority would belong to type II-ET.

The electrophysiological results suggest a subdivision among our patients on the basis of the elec- 
Table 3 Therapeutic results in the patients which were followed up

\begin{tabular}{|c|c|c|c|}
\hline & No & Propranolol & Primidone \\
\hline Group A & $\begin{array}{r}1 \\
3 \\
4 \\
5 \\
8 \\
9 \\
10 \\
11 \\
12 \\
13 \\
14 \\
17 \\
18 \\
19 \\
22 \\
23 \\
26 \\
29\end{array}$ & $\begin{array}{l}++ \\
+ \\
++ \\
+ \\
++ \\
++ \\
+ \\
++ \\
+ \\
++ \\
++ \\
++ \\
+ \\
+ \\
++ \\
++ \\
++ \\
++\end{array}$ & $\begin{array}{l}- \\
- \\
- \\
- \\
- \\
- \\
- \\
- \\
- \\
- \\
- \\
- \\
- \\
- \\
- \\
-\end{array}$ \\
\hline Group B & $\begin{array}{l}30 \\
32 \\
34 \\
36 \\
37 \\
38 \\
39 \\
40 \\
41 \\
42 \\
43 \\
44 \\
45\end{array}$ & $\begin{array}{l}0 \\
0 \\
0 \\
0 \\
0 \\
0 \\
+ \\
0 \\
0 \\
0 \\
0 \\
0 \\
+\end{array}$ & $\begin{array}{l}- \\
- \\
- \\
++ \\
+ \\
++ \\
++ \\
++ \\
+ \\
++ \\
++ \\
0 \\
++\end{array}$ \\
\hline
\end{tabular}

0, no improvement; + , moderate improvement; ++ , marked improvement: - , not given.

tromyographic tremor pattern and the occurrence of abnormal reflex responses.

The major group A had either activation of the antigravity muscle alone or coactivation of antagonists. This feature was described by Shahani and Young $^{7}$ and confirmed by others. ${ }^{8-10}$ The minor group B had alternating tremor bursts in antagonists. Shahani and Young found this only in $5 \%$ of their patients and therefore they suggested that they would probably develop Parkinson's disease. Several recent reports did not support this view. Particularly Sabra and Hallett ${ }^{10}$ declared the isolated tremor of the postural type with alternating activity to be a subgroup of essential tremor. From our own experience it seems most unlikely that there is a relation to Parkinson's disease. Some of our patients (group B) suffered from tremor for 10 to 15 years and in one case even 40 years without developing symptoms of Parkinsonism.

It has been found occasionally ${ }^{69}$ that the reciprocal alternating and the synchronous pattern in antagonists can be found at different times in one and the same patient. Our data confirm this finding under action conditions but we did not find a change under our defined postural condition. On the other hand we have seen severe alternating Parkinsonian tremor persisting during movement which changed its activation pattern under action conditions to synchronous activity without frequency changes. Despite these findings which are not well understood, there is obviously general agreement that some patients have a preponderant alternating pattern whereas others have a synchronous one. Therefore we agree with the suggestion that tremor should not be classified solely on basis of these phase relations. ${ }^{6}$ Neither should this phenomenological property of essential tremor be excluded from tremor classification as it may help to differentiate tremor subgroups among other criteria.

As a second criterion the reflex pattern to median nerve stimulation may distinguish the different forms of essential tremor. The normal pattern with H-reflex and LLR II is found only in group A patients. Group $B$ patients displayed an enhanced LLR I. It might be contested whether LLR I and LLR II are really two separate reflexes or if they are two extreme values of a uniform reflex. Several arguments are in favour of the first hypothesis. Firstly in normals LLR I never occurs without LLR II. A second argument may be derived from latency considerations: assuming that the shorter latency of LLR I would be the result of enhanced spatial and temporal summation of a common reflex pathway for LLR I and LLR II, it has to be expected that the latency would exhibit a continuous distribution between LLR I and LLR II. However, the distribution is clearly bimodal (fig 3) which is in favour of two separate reflex pathways. Moreover it has been demonstrated earlier by Marsden et al, ${ }^{11}$ that sudden stretch of flexor pollicis longus also evokes two different late reflex responses termed $\mathrm{A}$ and $\mathrm{B}$, whose latencies are compatible with the LLR I and II of our experiments.

The demonstration of two different populations among essential tremor patients concerning the long latency reflex is a new finding. Stretch evoked long latency reflex on flexor pollicis longus in patients with essential tremor $^{312}$ did not reveal any significant abnormalities from normals. However, the investigated muscle and the method of eliciting these reflexes were different from those in the present experiments and their population of patients could probably entirely correspond to group A according to our classification. Nevertheless future experiments have to clarify whether the LLR I enhancement can also be demonstrated in other muscles and with other methods.

The finding of an enhanced LLR I gives rise to the question, whether different pathophysiological mechanisms underly those two tremor types. Essential tremor is believed to be related to a central generator $^{313}$ but abnormal reflexes could equally account for tremor genesis. Computer simulations of 
a tremor model $^{14}$ suggested that long latency reflexes might contribute to tremor because their inherent reflex delay could maintain an ongoing rhythmic oscillation. The present investigation has identified a subgroup among essential tremor with abnormal reflex activity. However, to which extent abnormal reflexes are involved in tremor genesis has to be clarified by further experiments.

The present data about the pharmacological responsiveness of our patients confirm an earlier report. ${ }^{10}$ The patients of group A who had been treated with propranolol responded moderately or markedly but most of the patients in group B did not. The latter finding is in line with Sabra and Hallett's observation in 12 patients with alternating tremor bursts in antagonists ${ }^{10}$ who could correspond to our group B patients. This differential responsiveness may serve as a further argument which separates group B from group A patients. Primidone is meanwhile well established in the therapy of essential tremor. ${ }^{1516}$ As only patients of group B have been treated with primidone we cannot comment whether its beneficial effect is limited to patients of group B only. However, the results justify a double-blind, cross-over study whether the proposed electrophysiological classification predicts therapeutic responsiveness for the two drugs.

In conclusion the present study favours two forms of essential tremor with the following characteristics:

Type A: preponderant synchronous activity in antagonists under postural conditions, normal LLR, tremor frequency $5 \cdot 5-10 \mathrm{~Hz}$.

Type B: preponderant alternating activity in antagonists under postural conditions, abnormal LLR with an enhanced LLR I, tremor frequency $5 \cdot 5-8 \mathrm{~Hz}$.

It seems that type $A$ patients respond to propranolol whereas type B patients do not, but may respond to primidone.

The authors thank Professor M Hallett for helpful comments on the manuscript.

Supported by the Deutsche Forschungsgemeinschaft (SFB 325).

\section{References}

1 Rautakorpi I, Takala J. Martilla RJ, Sievers K, Rinne UK. Essential tremor in a Finnish population. Acta Neurol Scand 1982;66:58-67.

2 Rajput AP, Beard KPOM, Kurland LT. An epideminiologic survey of essential tremor in Rochester. Neurology 1982;32:128-34.

3 Marsden CD, Obeso JA, Rothwell JC. Benign essential tremor is not a single entity. In: Yahr MD, ed. Current concepts of Parkinson's disease and related disorders. Amsterdam: Excerpta medica 1983:31-46.

4 Deuschl G, Schenck E, Lücking $\mathrm{CH}$. Long-latency Reflexe und EMG-Polygraphie bei Patienten mit Parkinson- und essentiellem Tremor. Z EEG-EMG 1985;16:35-6.

5 Deuschl G, Schenck E, Lücking CH. Long-latency responses in human thenar muscles mediated by fast conducting muscle and cutaneous afferents. Neurosci Lett 1985;55:361-6.

6 Elble RJ. Physiologic and essential tremor. Neurology 1986;36:225-31.

7 Shahani BT, Young RR. Physiological and pharmacological aids in the differential diagnosis of tremor. J Neurol Neurosurg Psychiatry 1976;39:772-83.

8 Erbel F. Pathophysiologie des Tremors. Urban \& Schwarzenberg, 1979.

9 Larsen TA, Calne DB. Essential tremor. Clin Neuropharmacol 1983;6,3:185-206.

10 Sabra AF, Hallett $M$. Action tremor with alternating activity in antagonist muscles. Neurology 1984;34:151-6.

11 Marsden CD, Merton PA, Morton HB, et al. Automatic and voluntary responses to muscle stretch in man. In: Desmedt JE, ed. Progress in Clinical Neurophysiology. Cerebral motor control in man: long loop mechanisms. Basel: Karger, 1978:167-77.

12 Kachi T, Berardelli A, Day BL, Rothwell JC, Marsden CD. Pathophysiology of essential tremor. J Neurol 1985; Suppl Vol 232:134.

13 Lee RG, Stein RB. Resetting of tremor by mechanical pertubations: a comparison of essential tremor and parkinsonian tremor. Ann Neurol 1981;10:523-31.

14 Stein RB, Öguztöreli MN. Reflex involvement in the generation of tremor and clonus. In: Desmedt JE, ed. Physiological Tremor, Pathological Tremor and Clonus. Basel: Karger, 1978:28-50.

15 Findley LJ, Calzetti S, Cleeves L. Primidone in essential tremor. In: Findley LJ, Capildeo R, eds. Movement Disorders: Tremor. London: Macmillan Press Ltd, 1984:271-82.

16 Findley LJ, Cleeves L, Calzetti S. Primidone in essential tremor of the hands and head: a double blind controlled clinical study. J Neurol Neurosurg Psychiatry 1985;48:911-5. 